\title{
IMAGING IN ENDOCRINOLOGY \\ The use of functional MRI to study the endocrinology of appetite
}

\author{
Victoria Salem and Waljit S Dhillo \\ Section of Investigative Medicine, Imperial College London, Hammersmith Hospital Campus, \\ 6th Floor Commonwealth Building, Du Cane Road, London W12 0NN, UK
}

Correspondence should be addressed to W S Dhillo

Email

w.dhillo@imperial.ac.uk

\begin{abstract}
In the present review article, we summarise current thinking about the neuroendocrinology of appetite and feeding behaviour. We discuss how the homeostatic control of energy balance, wherein the hypothalamus orchestrates food intake and energy expenditure in response to peripheral signals about nutritional status, can be easily overridden by the powerful reward value of food. We focus on how functional magnetic resonance imaging has shed light on our understanding of the way hormones can interact with the brain to modulate appetite.
\end{abstract}

\section{Introduction}

The global obesity epidemic continues to reach ever greater proportions. Behavioural, diet and exercise programmes have limited success rates (1) and the number of safe and effective weight loss drugs has dwindled as the centrally mediated, dangerous side effects of these medications have become evident (2). It is hoped that a thorough understanding of the biology of food intake will lead to more successful prevention and treatment interventions.

Simply framed, obesity is the result of an imbalance between calories consumed and those expended. It has long been understood that hormones have a part to play in controlling energy homoeostasis. They play an important role in transmitting information to the brain about peripheral nutrient availability and fat stores, and they therefore affect appetite and energy expenditure and influence body weight (3). However, humans can easily override hormonal satiety signals when confronted with appetising foods associated with a reward value. This so-called non-homeostatic drive to eat, or cue reactivity, in our modern obesogenic environment is thought to have been a major contributor to the rapid rise in obesity rates in the past half century (4). More recently, emerging evidence has suggested that the complex reward, motivational and restraint behaviours associated with feeding may themselves be influenced by the

\section{Invited Author's profile:}

W S Dhillo is Professor of Endocrinology \& Metabolism and Consultant Endocrinologist at Imperial College, London. His research has focused on understanding the neuroendocrine mechanisms which are important in the regulation of food intake. The regulation of body weight is complex and requires the control of both food intake and energy expenditure. Gut hormones can powerfully reduce appetite and increase energy expenditure. Professor Dhillo's research investigates the mechanisms by which gut hormones mediate their effects.

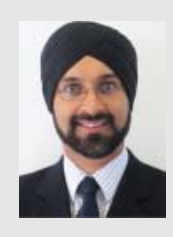

(C) 2015 European Society of Endocrinology Printed in Great Britain
Published by Bioscientifica Ltd. 
hormonal milieu, and the study of the neuroendocrinology of appetite has led us from the hypothalamus to the corticolimbic system and beyond. The system was initially arbitrarily divided into the homeostatic and non-homeostatic (or reward-driven) control of feeding behaviour, but it should now be thought of as a complicated neurohormonal appetite network. This is schematically illustrated in Fig. 1.
Functional magnetic resonance imaging (fMRI) is our most powerful tool to safely and non-invasively study the brain processes that underlie human appetitive behaviour. The past decade has witnessed an explosion in the number of neuroimaging studies in this field. In the present review, we will focus particularly on how fMRI has informed our understanding of the neuroendocrinology of appetite control.

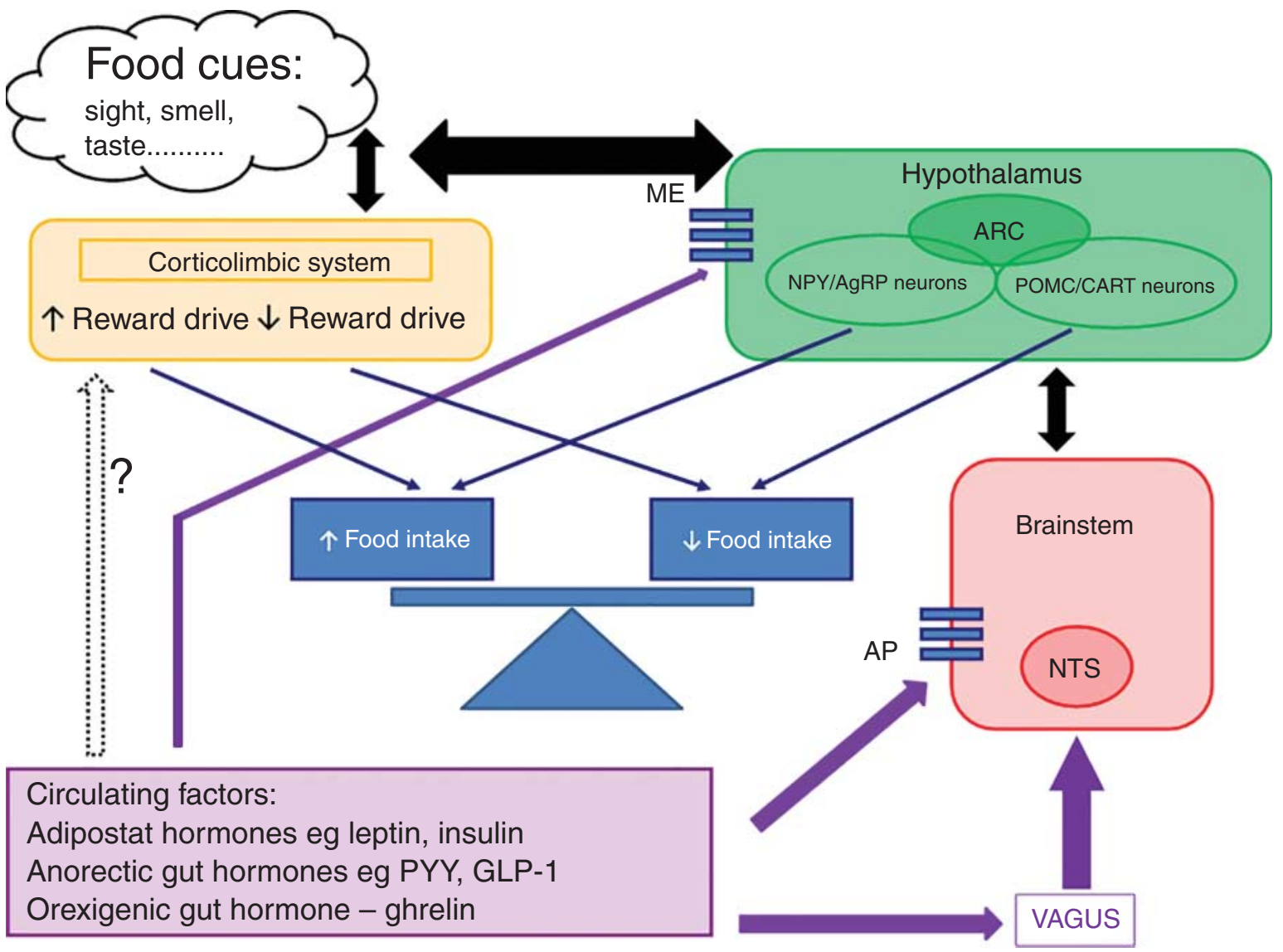

\section{Figure 1}

Schematic of the neuroendocrine network of appetite control. Circulating adipostat hormones, which signal long-term energy stores, and prandially released gut hormones, which signal short-term nutrient status, are able to directly influence neuronal activity in key brain areas involved in energy homoeostasis, the brainstem and the hypothalamus. Circulating gut hormones (such as peptide YY (PYY), glucagon-like peptide 1 (GLP-1) and ghrelin) modulate gastrointestinal vagal sensory input into the brainstem. They may also directly access the brainstem's nucleus of the solitary tract (NTS) and the hypothalamic arcuate nucleus (ARC) via deficient areas in the blood-brain barrier (the area postrema (AP) and the median eminence (ME)). Two major subpopulations of neurons in the ARC influence appetite. Those which co-express neuropeptide $Y$ (NPY) and agouti-related protein (AgRP) are orexigenic, whereas those which co-express pro-opiomelanocortin (POMC) and cocaine- and amphetamine-regulated transcript (CART) are anorexigenic. There are also hedonic reward drives orchestrated within the corticolimbic brain structures and superimposed on this homoeostatic system of appetite control; these reward drives are stimulated by sensory cues and possibly directly modulated by circulating factors. 


\section{Hormones and appetitive behaviour}

The characterisation of the CNS effects of leptin, an adipokine that conveys information about the body's fat stores, circulating levels of which drop with fasting to trigger a concerted CNS response in order to conserve body weight, has revolutionised our understanding of energy homoeostasis (5). Gut hormones such as peptide YY (PYY) and glucagon-like peptide 1 (GLP-1), which are postprandial signals of satiety, cholecystokinin (CCK), which is capable of modulating vagal afferent activity, and ghrelin which is released from the stomach when fasted to act as a powerful meal-inducer, have also been subject to intense research (3). The vast majority of this research has centred on the interaction of these hormones with the hypothalamus, which coordinates neural (largely vagal) and hormonal signals about the body's energy status into the reciprocal modulation of appetite (food-seeking behaviour) and metabolism (energy expenditure) (6).

Understanding the non-homeostatic control of food intake (and ultimately how hormones may also directly interact with these processes) requires an understanding of reward processing, memory, learning and executive behaviours, such as restraint. These concepts are reviewed in a scholarly manner and in much greater detail elsewhere $(6,7)$, but for the purposes of contextualising the modern understanding of human eating behaviour, they are briefly discussed here.

Human imaging studies related to food reliably demonstrate activity in brain regions implicated in sensory processing (the insula and precentral gyrus), reward processing (the nucleus accumbens, ventral striatum and orbitofrontal cortex (OFC)), memory, learning and emotion (the hippocampus and amygdala) and executive function (the prefrontal cortex) $(8,9)$. The corticolimbic reward system is thought to have evolved to promote behaviours which aid survival, such as eating (10). Dopaminergic transmission in these brain regions is involved in multiple processes that are connected to these concepts, such as pleasure sensation, the reinforcement of learning, incentive salience and motivation. As a cautionary note, many of these processes are referred to ambiguously in the obesity literature, which partly reflects the relative infancy of this field of study (11).

Investigators such as Berridge have separated the neurological and anatomical elements of reward into 'liking' (the intrinsic pleasurable sensation of a stimulus) and 'wanting' (which begins to describe how reward processing influences behaviour) (12). The salience of an object (or image or situation) is how much it stands out or how emotionally arousing it is. Saliency detection is important for focusing the brain's limited cognitive resources on the most pertinent environmental stimuli, and it facilitates learning. Incentive salience should be distinguished from more conscious forms of desire, but doing so requires the definition of a goal or expected outcome (13). In addition, exposure to food cues can trigger other, higher cognitive (including inhibitory) processes, such as restraint or the willpower to resist fatty foods in order to achieve the greater, long-term gain of maintaining a healthy weight $(10,14)$.

Past experience with specific foods is an important contributor to the reward process. Learning entrains conditioned stimuli which predict the availability and motivational value of reward and in time themselves become triggers of incentive salience $(15,16)$. In humans, simply imagining food can elicit cravings, and vivid imagery of food is a particularly potent cue for activation of the reward system (17). Furthermore, the activity of the hippocampus, a brain area that has been well described for its involvement in memory, is modulated in response to food cues (18).

Rodent models confirm a structural and functional connection between the hypothalamus, hippocampus and corticolimbic systems (19). fMRI has allowed us the opportunity to probe this interface between neuroendocrinology and psychology in humans. The field continues to grow. In the present review, we hope to introduce readers from an endocrine background to this intriguing and important field of study.

\section{Basic principles of functional MRI as applied to appetite studies}

fMRI utilises adaptations of the classical MRI technique, such that brain activity can be examined rather than structure alone. Virtually all fMRI studies rely on a measure called blood-oxygenation-level dependent (BOLD) contrast, which is based on the fact that oxygenated and deoxygenated blood possess different magnetic properties. Increased neuronal activity in the brain elicits a local haemodynamic response, which causes an increase in blood flow that results in local magnetic field disturbances that can be detected on a $2^{*}$-weighted imaging protocol (20). Temporal resolution is limited by the short delay between neuronal activation and the measurable haemodynamic response (21).

fMRI image reconstruction centres on measured signals from spatially defined brain areas fitted against a theoretical haemodynamic response function timed 
against an applied stimulus or cue. The stimulus can be the oral delivery of a sweet-tasting drink, but the most common experimental paradigm in the field of appetite imaging is to present the subject with conditioned cues, which are usually pictures of food.

A subtraction analysis can be performed to see whether the difference in regional brain activation between viewing images of food or non-food is altered in different conditions (e.g. the fasted vs the fed state, or a contrast of contrasts). These experiments are also commonly performed along with reported personality or behaviour scoring systems, which are subjective sensory ratings or metabolic measurements (that are then correlated with measured neuronal activations) $(22,23)$.

Ultimately, most fMRI studies start by looking at whole-brain activation patterns to pinpoint the areas of brain activity that are significantly modulated by the study variable in question. To improve the power to detect significantly modulated brain activity, the hypothalamus and corticolimbic brain structures are often used as predefined regions of interest (ROIs) in neuroimaging studies based on previous understanding about the role they play in the processing of appetite, reward, memory or other behaviours. This is because a smaller number of predefined ROIs decreases the degrees of freedom in the comparative statistical analysis stage.

\section{A word about reproducibility}

Because imaging the hypothalamus (which is small in size and prone to suffer from image artefact because of its proximity to the sinuses) is technically challenging, and also because cue-driven processing plays such an important role in human eating behaviour, neuroimaging studies in this field have tended to concentrate on the corticolimbic brain areas associated with reward and motivation.

fMRI relies on the measurement of small signal changes among large, inter-subject differences. Appetite pathways, and the reward system in general, are intricately tuned by numerous genetic, developmental, psychological, environmental and hormonal influences. Factor into this the statistical problem of performing multiple comparisons between individual or even clusters of voxels and variable experimental paradigms, and it is unsurprising that a recent meta-analysis reported that concurrence between appetite imaging studies was moderate: at best, $41 \%$ (seven out of 17 studies) concurred on significantly activated voxel clusters for the contrast between food and non-food pictures (24). In response to this, new databases of food images (of standardised luminosity, complexity and known calorie content) are becoming available with the hope of facilitating comparability across appetite neuroimaging studies (25). Conversely, some modern experimental paradigms have tried to account for the heterogeneity in personal food-cue reactivity by tailoring the stimuli used to elicit appetitive neuronal responses to pre-assessed personal preferences (26).

\section{The fed vs the fasted brain}

Imaging studies that investigate appetite regulation in human subjects have confirmed the notion that images of food can trigger the brain's reward system and that the potency of this trigger is greater in the fasted state.

In one of the earliest fMRI studies of its type, LaBar et al. (27) scanned nine healthy subjects after an $8 \mathrm{~h}$ fast and then performed a post-meal scan $1 \mathrm{~h}$ later. A follow-up study was performed on eight subjects who were fed a meal before the first of the two scans. Subtraction analysis of food minus non-food images revealed a satiety-induced reduction in activity in the amygdala and parahippocampal gyrus, although this early study was underpowered to detect similar satiety-induced changes in other a prioridefined corticolimbic ROIs. Following this, Killgore et al. scanned fed volunteers while they viewed high-calorie, low-calorie and non-food pictures. The authors reported food-responsive regions of the brain, that is, areas where food images caused greater activation than non-food images did, including the amygdala, hippocampus and ventromedial prefrontal cortex (28). Another early fMRI study by O'Doherty et al. (8) reported that expectation of a pleasant taste (in subjects trained to expect the oral delivery of a sweet taste following a given visual cue) produced activation in the amygdala, striatum and OFC in a different pattern from that observed following the actual reward receipt.

More recently, Fuhrer et al. in 2008 studied 12 healthy male volunteers using two separate scanning sessions one after overnight fasting and the other immediately after a large meal. They particularly noted significantly enhanced activity within the OFC when the subjects were hungry (29). Siep et al. (30) reported increased activity in the amygdala and OFC in response to viewing high-calorie vs low-calorie food images but only when their subjects were fasted and actively evaluating the food image (but not when their attention was drawn elsewhere).

fMRI studies often also attempt to correlate brain signal changes with other variables, such as subjective ratings of appetite or circulating hormone levels. In 2009, Goldstone et al. were able to tease out areas of the brain 
that were activated in response to high-calorie rather than low-calorie foods, including the ventral striatum, amygdala, anterior insula and medial and lateral OFC. The selective modulation of these areas by high-calorie foods was amplified in the fasting state, and, even more specifically, the change in (subjectively reported) appeal bias towards high-calorie foods was positively correlated with OFC activation (31). Very recently, Stice et al. (32) showed that the duration of caloric deprivation correlated positively with activation in various corticolimbic reward centres (e.g. the anterior cingulate cortex, OFC, putamen and precentral gyrus).

\section{The obese vs the lean brain}

There is evidence from imaging studies that obese subjects exhibit greater reactivity of reward circuitry in response to the consummation of palatable foods. This fits into the theory that reward 'hypersensitivity' may be a risk factor for overeating. A commonly used model for understanding eating behaviour is to think of a 'reward' as comprising two phases, anticipatory and consummatory, and a number of fMRI studies have been designed to separate out neural responses in each of these conditions $(9,32,33)$.

Using positron emission tomography (PET) scanning to measure regional cerebral blood flow ( $\mathrm{rCBF}$, which is the same marker of neuronal activity quantified by fMRI), DelParigi et al. studied the differences between the responses of obese and lean subjects to the receipt of a liquid meal after a prolonged fast. Differences in rCBF were observed in several regions of the brain, and they included greater activation in the middle dorsal insula and midbrain and greater decreases in the posterior cingulate, temporal and orbitofrontal cortices in obese as compared to lean individuals (34). The same group also demonstrated that that the dorsal insula and posterior hippocampus remained abnormally responsive to the consumption of food in previously obese as compared to lean individuals, which led them to the conclusion that these abnormal responses may increase the risk for obesity (35).

Visual food cues can also evoke significantly greater reward system activation in the obese group in several brain regions implicated in food reward. In 2007, Rothemund et al. scanned fasted volunteers: 13 obese and 13 normal weight women. They found that BMI positively predicted BOLD activation of the caudate, putamen, anterior insula and lateral OFC when the subjects were viewing high-calorie food images (36). Stoeckel et al. (37) also showed that pictures of highcalorie foods (vs non-food) produced greater activation in 12 obese subjects as compared to 12 lean controls in the ventral and dorsal striatum, insula, anterior cingulate cortex, amygdala and OFC. Scharmuller et al. (38) repeated the observation that obese participants display greater activation of the insula than do lean participants during the passive viewing of food cues.

Burger and Stice performed a prospective study to tackle the question of whether reward hyper-responsiveness to food cues actually led to overeating. They scanned 35 women and found that repeated exposure to a cue that predicted milkshake receipt caused an increase in signal in the caudate. Conversely, repeated exposure to the milkshake itself caused a decrease signal in the putamen. Over a 2-year subsequent follow-up period, BMI gain correlated with both the size of the signal increase that occurs in response to cue-reward learning and the size of the signal decrease that occurs with repeated exposure to a sweet taste (39). In a non-prospective study, Killgore et al. (40) reported that subjective scores of motivational status correlated with corticolimbic food responsiveness and that this measure could be independently used to predict BMI in their female cohort.

It should be noted that there are also many examples of studies reporting on the hypo-responsiveness of certain brain areas in response to food cues in the obese. Cornier et al. (41) recently showed that the measured neuronal response to the expectation of sweet taste (but not the response to taste receipt) is down-regulated in individuals prone to weight gain and obesity. A recent meta-analysis by Brooks et al. (42) suggested that the areas linked to the evaluation of rewarding stimuli appear most consistently activated in response to images of food in those who are obese. However, they also noted that a reduced activation in brain regions associated with cognitive control and awareness of bodily sensations is often reported in the obese, which suggests that a weakened self-control system or hypo-sensitivity to satiety and discomfort signals after eating may also contribute to those who are prone to overeat (42). For example, Le et al. (43) reported reduced activation in the prefrontal cortex, an area that has been described as playing a central role in the inhibition of inappropriate behaviours, in obese women in response to a meal as compared to lean or formerly obese women.

There is also some evidence that obese individuals have reduced consummatory responses in parts of the reward network, such as the dorsal striatum $(9,44)$. A major neurotransmitter in the reward network is dopamine, and tracer studies have suggested that dopamine is indeed released in the dorsal striatum of normal subjects in response to the sight and smell of food (45). 
Furthermore, feeding is associated with dopamine release in the dorsal, but not the ventral, striatum, and the amount of dopamine released correlates with the degree of experienced pleasure (46). Thus, it has been argued that one explanation for some of the reduced dorsal striatal consummatory responses measured in certain MRI studies of feeding may be hypo-dopaminergic functioning (47). As such, obese people are posited to require more caloric intake for a given level of a rewarding experience, and food is used as a form of self-medication to boost hedonic capacity. In line with this, nuclear imaging studies have revealed a reduction in striatal dopamine receptor density in overweight subjects $(48,49)$. Alternatively, these findings have been explained as being the result of down-regulation resulting from overeating (50), in other words, as a phenomenon of tolerance that mirrors models of drug dependence (51). This competing theory suggests that individuals at risk of obesity actually have a hyperreactive reward system in the first place. Such individuals experience greater reward from food intake, which drives overeating and subsequently results in a tachyphylaxis of dopamine signalling in response to food intake (52), thereby driving greater caloric intake for a given 'hit'. The concept of obesity as a consequence of food addiction has attracted intense debate in the literature, and readers are referred to these excellent reviews for a broader picture of the argument $(11,53)$.

In summary, there is emerging evidence that obese individuals have abnormal reward centre activity relative to lean individuals, although many more well-planned, longitudinal studies are required to disentangle cause from effect in the case of overeating. In general, obese individuals tend to have hyper-responsive reward activation patterns in both anticipatory and consummatory paradigms, with corresponding evidence of weaker activation in the brain areas associated with executive functions, such as restraint. However, there is also evidence that obese people may have a hypo-dopaminergic striatum that drives overconsumption.

\section{The gut-brain axis}

We are all able from personal experience to appreciate how food tastes better when we are hungry. fMRI studies have revealed some of the neuroanatomical correlates of this process. What are the peripheral signals that converge on the corticolimbic reward centres to modulate their foodcue responsivity? During the past decade, a number of fMRI studies have switched their attention to the neuroendocrinology of appetite, investigating how peripheral hormones which regulate energy homoeostasis also interact with food-responsive brain regions.

Leptin plays a major role in signalling to the brain information about the body's energy stores. Circulating concentrations of this fat-derived hormone increase with adipose tissue mass. Declining leptin levels indicate the onset of negative energy balance, which acts as a powerful hypothalamic signal to increase food intake. Leptin deficiency across species induces morbid obesity fuelled by hyperphagia. Human cases of severe obesity secondary to leptin deficiency are extremely rare but, when identified, have been successfully treated with recombinant leptin, with the principal effect being a reduction in appetite (54). In two congenitally leptin-deficient human subjects, daily subcutaneous leptin replacement reduced BOLD fMRI activation when viewing food vs non-food images in the nucleus accumbens, caudate, putamen and globus pallidus (55). These findings have been recently extended to healthy subjects, in whom there is positive correlation between plasma leptin concentration and fMRI signal in the ventral striatal regions during the presentation of visual food cues (56). Early theories suggested that leptin interacted with hypothalamic neurons to govern ingestive behaviour, but these later studies opened up the possibility that circulating hormones were able to modulate higher cortical function as well.

Leptin acts as a signal of long-term energy stores, but energy consumption can also be centrally modulated on a meal-by-meal basis by peptide hormones released from the gut. The control of meal size is largely determined by the onset of satiety. There are a number of postprandially secreted gut hormones, particularly the L-cell-derived PYY and GLP-1, which enhance the sensation of satiety. Conversely, stomach-derived ghrelin stimulates ingestive behaviour, with peak circulating levels occurring in the fasting state. In the same year that the effects of leptin on corticolimbic fMRI signal were first reported, Batterham et al. (57) showed that in fasted, lean individuals, energy intake at lunch was predicted by the modulation of an OFC signal following PYY ${ }_{3-36}$ infusion. When the subjects received saline infusion, subsequent (lunch-time) food intake better correlated with changes in hypothalamic signal. It was postulated that the presence of $\mathrm{PYY}_{3-36}$ switches the regulation of food intake from a homeostatic brain region (the hypothalamus) to a non-homeostatic one (the OFC), although this study looked at resting-state BOLD signals as opposed to the contrast between food cue-activated signals described elsewhere in the present review. In 2011, we reported the effects of single and combination doses of the anorectic gut hormones $\mathrm{PYY}_{3-36}$ 
and GLP-1 ${ }_{7-36}$ amide that were given to fasted volunteers as compared to the effects off saline in the fasted state or saline in the fed state (that is, just after a large breakfast). In the fed state (i.e. after breakfast), volunteers ate significantly less at lunch than they did when they had remained fasted from the previous night. Administering combination $\mathrm{PYY}_{3-36}$ or GLP-1 ${ }_{7-36}$ amide to our fasted subjects also reduced the amount of food they consumed at lunch to the same extent as if they had eaten breakfast. During the course of each of the interventions described earlier, we performed BOLD fMRI scanning to measure differences in relative (food minus non-food) activation in several preselected corticolimbic ROIs. In all of our a prioridefined ROIs (the caudate, puamen, nucleus accumbens and OFC), the signal contrast when viewing images of food as compared to non-food was attenuated in the fed state, and this was recreated in the fasted state when anorectic gut hormones were infused. In other words, the hyperresponsivity of the corticolimbic reward system to visual food cues that occurred when our subjects were hungry was dampened when we administered GLP-1 and PYY to them (58). In a study that mirrored these findings, Malik et al. (59) demonstrated that the hypo-responsiveness of the reward system to food cues when full can be reinvigorated with the delivery of the appetite hormone ghrelin. Goldstone et al. (60) confirmed that the pattern of reward-centre responsivity to food cues when fasted was indistinguishable from that induced by ghrelin, which added weight to the hypothesis that circulating gut hormones are responsible for the physiological modulation of reward reactivity that was first measured in the very earliest fed vs fasted MRI appetite studies. In an interesting twist to this experimental paradigm, Van Oudenhove et al. (61) recently showed that gastric fatty acid infusion (which stimulated an endogenous release of the gut hormone CCK) attenuated both the behavioural and neural responses to sad emotion induction (and that this effect was blocked by a CCK receptor antagonist).

More recent studies have attempted to tease out the hormone-brain interactions in more detail. Kroemer et al. (62) showed that circulating ghrelin levels actually correlate positively with food-cue responsivity as measured by fMRI in both homeostatic and non-homeostatic regions. $\mathrm{Li}$ et al. reported that ingestion of liquid meals with different macronutrient contents all reduced BOLD activity in the middle insula, thalamus, parahippocampal cortex, caudate, amygdala and lateral OFC. In the insula and OFC, ghrelin levels positively correlated and GLP-1 levels negatively correlated with the measured postprandial changes in BOLD activity (63). A recent confirmation of the effects of GLP-1 signalling on brain appetitive responses came from van Bloemendaal et al., who reported that obese vs lean subjects had increased activation in the insula, OFC and NAc in response to food cues and that IV exanatide (a long-acting GLP-1 agonist) reduced these responses. They were able to demonstrate a strong correlation between the dampening of this corticolimbic food-cue reactivity with subsequent food intake and also showed that these GLP-1 mediated effects were indeed abolished with the co-administration of the GLP-1 receptor antagonist exendin 9-39 (64). GLP-1 receptor analogues are now gaining licence as pharmacotherapies for obesity, even in the absence of diabetes.

In summary, the detailed mechanisms underlying this interaction between reward, emotion and peripheral hormonal signals of energy status remain to be elucidated. Imaging data in this field so far points to the suggestion that circulating (meal-related) gut hormones and (fatrelated) adipokines are able to modulate the conditioned relationships between environmental food cues and reward drive.

\section{Bariatric surgery}

Bariatric surgery is the most effective current treatment for sustained weight loss and the complications of obesity. The mechanisms behind the weight loss seen following Roux-en-Y gastric bypass (RYGB) surgery remain to be fully elucidated. It is now clear that the profound and sustained weight loss achieved with this procedure is not related simply to the malabsorption of nutrients but rather to a constellation of neurohormonal effects. These include elevated post-operative levels of endogenous anorectic gut hormones such as PYY and GLP-1, increases in energy expenditure and metabolic rate, changes in taste preference away from calorie-dense foods, increased bile acid delivery to the ileum and changes in gut microbiota (65, 66). Recent studies imaging appetite in patients before and after surgical weight loss are important in helping us understand some of the CNS-mediated effects, with the potential translational application of identifying those patients who would respond best to bypass surgery.

In 2011, van de Sande-Lee et al. (67) performed fMRI studies on eight lean control subjects and 13 obese patients before and after RYGB, through which an average $30 \%$ reduction in body weight had been achieved. Using a BOLD fMRI paradigm that investigated areas of the brain whose signal intensity and pattern co-varied, they reported a high level of functional connectivity between the hypothalamus and the orbitofrontal and 
somatosensory cortices that was absent in obese subjects but was re-established with post-operative weight loss.

In the same year, a more conventional ROI-based study of ten obese female patients 1 month before and after RYGB revealed an attenuation of fMRI BOLD signals (when viewing high-calorie vs low-calorie food images) in the ventral tegmental area, ventral striatum, putamen, lentiform nucleus, posterior cingulate and dorsomedial prefrontal cortex following the bypass procedure (68). Reanalysis of this dataset showed that the post-operative changes in mesolimbic reward pathway response predicted changes in the subjective assessment of the desire to eat high- vs low-calorie foods (69).

Bruce et al. have recently reported nuanced changes in brain responsivity to food cues in patients who have undergone bariatric surgery as compared to those who lost weight through dieting alone. Dieters showed increased responses to food cues in the medial prefrontal cortex, a region associated with valuation. Patients who had undergone bariatric surgery showed increased responses to food cues in brain regions associated with higher-level perception. This is the first evidence in human subjects that the method of weight loss elicits different brain responses, and it may begin to explain why some approaches are more successful than others (70).

In a dopamine antagonist radiotracer study, Dunn et al. (71) demonstrated that 6 weeks after gastric bypass surgery, striatal dopamine binding increases, purportedly in relation to the reduction in appetite that is seen following the procedure (and is accounted for by the previously described observation that obesity is associated with hypodopaminergic reward functioning). Recently, Goldstone et al. reported that obese patients after RYGB had lower corticolimbic responses to food than did patients after gastric band surgery (which works to limit stomach size but has none of the other metabolic effects associated with bypass). RYGB patients had lower activation than band patients, particularly in response to high-calorie foods, in brain reward systems, including the OFC, amygdala, caudate and nucleus accumbens. This was associated with lower appeal ratings of high-calorie foods in RYGB as compared to band patients and BMI-matched un-operated control (72). Here, fMRI has provided a powerful tool to examine the brain effects of a gastrointestinal intervention - illustrating in colour the gut-brain axis at play.

\section{Conclusion}

fMRI has opened up a fascinating window into the complex brain processes that underpin overeating and obesity. It has also been the driver for extending our understanding of the neuroendocrine interface in humans. In the immediate future, imaging goals will include further understanding how the regulatory (hypothalamic) and reward (corticolimbic) centres interact with one another in the overall control of appetite and exactly how circulating hormones interact with these processes. A weight-matched study of the comparative effects of surgical and dietary weight loss is essential to further delineate the neural elements that are important to sustained weight loss. As our imaging techniques become more refined and powerful, pinpointing and detailing food-responsive circuitry will translate into the development of safer anorectic agents. From reducing the impact of billboard adverts for fast foods to selectively targeting the circuits that depress appetite but not mood, understanding the brain pathways that control food intake is of vital importance for finding new strategies to combat the world's leading health concern.

\section{Declaration of interest}

The authors declare that there is no conflict of interest that could be perceived as prejudicing the impartiality of the review.

\section{Funding}

V Salem is supported by a NIHR Clinical Lectureship. W S Dhillo is supported by a NIHR Career Development Fellowship.

\section{Acknowledgements}

The authors would like to thank the anonymous reviewers of the present article for their keen and insightful comments.

\section{References}

1 Dombrowski SU, Knittle K, Avenell A, Araújo-Soares V \& Sniehotta FF. Long term maintenance of weight loss with non-surgical interventions in obese adults: systematic review and meta-analyses of randomised controlled trials. British Medical Journal 2014348 g2646. (doi:10.1136/ bmj.g2646)

2 Salem V \& Bloom SR. Approaches to the pharmacological treatment of obesity. Expert Review of Clinical Pharmacology 20103 73-88. (doi:10.1586/ecp.09.54)

3 Chaudhri OB, Salem V, Murphy KG \& Bloom SR. Gastrointestinal satiety signals. Annual Review of Physiology 200870 239-255. (doi:10.1146/annurev.physiol.70.113006.100506)

4 Berthoud HR. The neurobiology of food intake in an obesogenic environment. Proceedings of the Nutrition Society 201271 478-487. (doi:10.1017/S0029665112000602)

5 Farooqi IS \& O'Rahilly S. 20 years of leptin: human disorders of leptin action. Journal of Endocrinology 2014223 T63-T70. (doi:10.1530/ JOE-14-0480)

6 Berthoud HR. Homeostatic and non-homeostatic pathways involved in the control of food intake and energy balance. Obesity $2006 \mathbf{1 4}$ 197S-200S. (doi:10.1038/oby.2006.308) 
7 Kanoski SE. Cognitive and neuronal systems underlying obesity. Physiology \& Behavior 2012106 337-344. (doi:10.1016/j.physbeh.2012.01.007)

8 O'Doherty JP, Deichmann R, Critchley HD \& Dolan RJ. Neural responses during anticipation of a primary taste reward. Neuron 200233 815-826. (doi:10.1016/S0896-6273(02)00603-7)

9 Stice E, Spoor S, Bohon C, Veldhuizen M \& Small D. Relation of reward from food intake and anticipated food intake to obesity: a functional magnetic resonance imaging study. Journal of Abnormal Psychology 2008 117 924-935. (doi:10.1037/a0013600)

10 van den Bos R \& de Ridder D. Evolved to satisfy our immediate needs: self-control and the rewarding properties of food. Appetite $2006 \mathbf{4 7}$ 24-29. (doi:10.1016/j.appet.2006.02.008)

11 Salamone J \& Correa M. Dopamine and food addiction: lexicon badly needed. Biological Psychiatry 201373 e15-e24. (doi:10.1016/j.biopsych. 2012.09.027)

12 Castro DC \& Berridge KC. Advances in the neurobiological bases for food 'liking' and 'wanting'. Physiology \& Behavior 2014136 22-30. (doi:10.1016/j.physbeh.2014.05.022)

13 Robinson TE \& Berridge KC. Incentive-sensitization and addiction. Addiction 200196 103-114. (doi:10.1046/j.1360-0443.2001.9611038.x)

14 Bryant EJ, King NA \& Blundell JE. Disinhibition: its effect on appetite and weight regulation. Obesity Reviews 20089 409-419. (doi:10.1111/j. 1467-789X.2007.00426.x)

15 Berthoud HR \& Morrison C. The brain, appetite and obesity. Annual Review of Psychology 200859 55-92. (doi:10.1146/annurev.psych.59. 103006.093551)

16 Shin AC, Zheng H \& Berthoud HR. An expanded view of energy homeostasis: neural integration of metabolic, cognitive, and emotional drives to eat. Physiology \& Behavior 200997 572-580. (doi:10.1016/j. physbeh.2009.02.010)

17 Schur EA, Kleinhans NM, Goldberg J, Buchwald D, Schwartz MW \& Maravilla K. Activation in brain energy regulation and reward centers by food cues varies with choice of visual stimulus. International Journal of Obesity 200933 653-661. (doi:10.1038/ijo.2009.56)

18 Kanoski SE \& Davidson TL. Western diet consumption and cognitive impairment: links to hippocampal dysfunction and obesity. Physiology \& Behavior 2011103 59-68. (doi:10.1016/j.physbeh.2010.12.003)

19 Fulton S. Neurosystems linking corticolimbic and hypothalamic pathways in energy balance: view from the Chair. International Journal of Obesity 200933 S3-S7. (doi:10.1038/ijo.2009.64)

20 Ogawa S, Tank DW, Menon R, Ellerman JM, Kim SG, Merkle H \& Ugurbil K. Intrinsic signal changes accompanying sensory stimulation: functional brain mapping with magnetic resonance imaging. PNAS 199289 5951-5955. (doi:10.1073/pnas.89.13.5951)

21 Detre JA \& Wang J. Technical aspects and utility of fMRI using BOLD and ASL. Clinical Neurophysiology 2002113 621-634. (doi:10.1016/ S1388-2457(02)00038-X)

22 Beaver JD, Lawrence AD, van Ditzhuijzen J, Davis MH, Woods A \& Calder AJ. Individual differences in reward drive predict neural responses to images of food. Journal of Neuroscience 200626 5160-5166. (doi:10.1523/JNEUROSCI.0350-06.2006)

23 White MA, Whisenhunt BL, Williamson DA, Greenway FL \& Netemeyer RG. Development and validation of the food-craving inventory. Obesity Research 200210 107-114. (doi:10.1038/oby.2002.17)

24 van der Laan LN, De Ridder DT, Viergever MA \& Smeets PA. The first taste is always with the eyes: a meta-analysis on the neural correlates of processing visual food cues. NeuroImage 201155 296-303. (doi:10. 1016/j.neuroimage.2010.11.055)

25 Blechert J, Meule A, Busch NA \& Ohla K. Food-pics: an image database for experimental research on eaing and appetite. Frontiers in Psychology 20145 617. (doi:10.3389/fpsyg.2014.00617)

26 Jastreboff A, Sinha R, Lacadie C, Small DM, Sherwisn RS \& Potenza MN. Neural correlates of stress- and food cue-induced food craving in obesity: association with insulin levels. Diabetes Care 201336 395-492. (doi:10.2337/dc12-1112)
27 LaBar KS, Gitelman DR, Parrish TB, Kim YH, Nobre AC \& Mesulam MM. Hunger selectively modulates corticolimbic activation to food stimuli in humans. Behavioral Neuroscience 2001115 493-500. (doi:10.1037/ 0735-7044.115.2.493)

28 Killgore WD, Young AD, Femia LA, Bogorodzki P, Rogowska J \& Yurgelun-Todd DA. Cortical and limbic activation during viewing of high- versus low-calorie foods. NeuroImage 200319 1381-1394. (doi:10.1016/S1053-8119(03)00191-5)

29 Fuhrer D, Zysset S \& Stumvoll M. Brain activity in hunger and satiety: an exploratory visually stimulated FMRI study. Obesity $2008 \mathbf{1 6}$ 945-950. (doi:10.1038/oby.2008.33)

30 Siep N, Roefs A, Roebroeck A, Havermans R, Bonte ML \& Jansen A. Hunger is the best spice: an fMRI study of the effects of attention, hunger and calorie content on food reward processing in the amygdala and orbitofrontal cortex. Behavioral Brain Research 2009198 149-158. (doi:10.1016/j.bbr.2008.10.035)

31 Goldstone AP, Prechtl de Hernandex CG, Beaver JD, Muhammed K, Croese C, Bell G, Durighel G, Hughes E, Waldman AD, Frost G et al. Fasting biases brain reward systems towards high-calorie foods. European Journal of Neuroscience 200930 1625-1635. (doi:10.1111/ j.1460-9568.2009.06949.x)

32 Stice E, Burger K \& Yokum S. Caloric deprivation increases responsivity of attention and reward brain regions to intake, anticipated intake, and images of palatable foods. Addiction Biology 201318 855-862. (doi:10.1111/j.1369-1600.2012.00489.x)

33 Pelchat ML, Johnson A, Chan R, Valdez J \& Ragland JD. Images of desire: food-craving activation during fMRI. NeuroImage 200423 1486-1493. (doi:10.1016/j.neuroimage.2004.08.023)

34 DelParigi A, Chen K, Salbe AD, Reiman EM \& Tataranni PA. Sensory experience of food and obesity: a positron emission tomography study of the brain regions affected by tasting a liquid meal after a prolonged fast. NeuroImage 200524 436-443. (doi:10.1016/j.neuroimage.2004.08.035)

35 DelParigi A, Chen K, Salbe AD, Hill JO, Wing RR, Reiman EM \& Tataranni PA. Persistence of abnormal neural responses to a meal in postobese individuals. International Journal of Obesity and Related Metabolic Disorders 200428 370-377. (doi:10.1038/sj.ijo.0802558)

36 Rothemund Y, Preuschhof C, Bohner G, Bauknecht CH, Klingebiel R, Flor H \& Klapp BF. Differential activation of the dorsal striatum by highcalorie visual food stimuli in obese individuals. NeuroImage $2007 \mathbf{3 7}$ 410-421. (doi:10.1016/j.neuroimage.2007.05.008)

37 Stoeckel LE, Weller RE, Cook EW, Twieg DB, Knowlton RC \& Cox JE. Widespread reward-system activation in obese women in response to pictures of high-calorie foods. NeuroImage $2008 \mathbf{4 1}$ 636-647. (doi:10.1016/j.neuroimage.2008.02.031)

38 Scharmuller W, Ubel S, Ebner F \& Schienle A. Appetite regulation during food cue exposure: a comparison of normal-weight and obese women. Neuroscience Letters 2012518 106-110. (doi:10.1016/j.neulet. 2012.04.063)

39 Burger KS \& Stice E. Greater striatopallidal adaptive coding during cuereward learning and food reward habituation predict future weight gain. NeuroImage 201499 122-128. (doi:10.1016/j.neuroimage.2014.05.066)

40 Killgore WD, Weber M, Schwab ZJ, Kipman M, DelDonno SR, Webb CA $\&$ Rauch SL. Cortico-limbic responsiveness to high-calorie food images predicts weight status among women. International Journal of Obesity 201337 1435-1442. (doi:10.1038/ijo.2013.26)

41 Cornier MA, Shott ME, Thomas EA, Bechtell JL, Bessesen DH, Tregellas JR \& Frank GK. The effects of energy balance, obesityproneness and sex on the neuronal response to sweet taste. Behavioural Brain research 2014278 446-452. (doi:10.1016/j.bbr.2014.10.024)

42 Brooks SJ, Cedernaes J \& Schiöth HB. Increased prefrontal and parahippocampal activation with reduced dorsolateral prefrontal and insular cortex activation to food images in obesity: a meta-analysis of fMRI studies. PLoS ONE 20138 e60393. (doi:10.1371/journal.pone. 0060393)

43 Le DS, Pannacciulli N, Chen K, Salbe AD, Del Parigi A, Hill JO, Wing RR, Reiman EM \& Krakoff J. Less activation in the left dorsolateral 
prefrontal cortex in the reanalysis of the response to a meal in obese than in lean women and its association with successful weight loss. American Journal of Clinical Nutrition 200786 573-579.

44 Stice E, Spoor S, Bohon C \& Small DM. Relation between obesity and blunted striatal response to food is moderated by TaqIA A1 allele. Science 2008322 449-452. (doi:10.1126/science.1161550)

45 Volkow ND, Wang GJ, Maynard L, Jayne M, Fowler JS, Zhu W, Logan J, Gatley SJ, Ding YS, Wong C et al. Brain dopamine is associated with eating behavior in humans. International Journal of Eating Disorders 2003 33 136-142. (doi:10.1002/eat.10118)

46 Small DM, Jones-Gotman M \& Dagher A. Feeding-induced dopamine release in dorsal striatum correlates with meal pleasantness ratings in healthy human volunteers. NeuroImage 200319 1709-1715. (doi:10.1016/S1053-8119(03)00253-2)

47 Blum K, Braverman ER, Holder JM, Lubar JF, Monastra VJ, Miller D, Lubar JO, Chen TJ \& Comings DE. Reward deficiency syndrome: a biogenetic model for the diagnosis and treatment of impulsive, addictive, and compulsive behaviors. Journal of Psychoactive Drugs 2000 32 (Suppl i-iv) 1-112.

48 Wang GJ, Volkow ND, Logan J, Pappas NR, Wong C, Zhu W, Netsuil N \& Fowler NS. Brain dopamine and obesity. Lancet 2001357 354-357. (doi:10.1016/S0140-6736(00)03643-6)

49 Volkow ND, Wang GJ, Telang F, Fowler JS, Thanos PK, Logan J, Alexoff D, Ding YS, Wong C, Ma Y et al. Low dopamine striatal D2 receptors are associated with prefrontal metabolism in obese subjects: possible contributing factors. NeuroImage 200842 1537-1543. (doi:10.1016/j.neuroimage.2008.06.002)

50 Stice E, Yokum S, Blum K \& Bohon C. Weight gain is associated with reduced striatal response to palatable food. Journal of Neuroscience 2010 30 13105-13109. (doi:10.1523/JNEUROSCI.2105-10.2010)

51 Davis C, Strachan S \& Berkson M. Sensitivity to reward: implications for overeating and obesity. Appetite 200442 131-138. (doi:10.1016/ j.appet.2003.07.004)

52 Stice E, Yokum S, Zald D \& Dagher A. Dopamine-based reward circuitry responsivity, genetics, and overeating. Current Topics in Behavioral Neurosciences 20116 81-93. (doi:10.1007/7854_2010_89)

53 Ziauddeen $\mathrm{H} \&$ Fletcher PC. Is food addiction a valid and useful concept? Obesity Reviews 201314 19-28. (doi:10.1111/j.1467-789X. 2012.01046.x)

54 Farooqi IS, Jebb SA, Langmack G, Lawrence E, Cheetham CH, Prentice AM, Hughes IA, McCamish MA \& O'Rahilly S. Effects of recombinant leptin therapy in a child with congenital leptin deficiency. New England Journal of Medicine 1999341 879-884. (doi:10.1056/NEJM199909163411204)

55 Farooqi IS, Bullmore E, Keogh J, Gillard J, O’Rahilly S \& Fletcher PC. Leptin regulates striatal regions and human eating behavior. Science 2007317 1355. (doi:10.1126/science.1144599)

56 Grosshans M, Vollmert C, Vollstadt-Klein S, Tost H, Leber S, Bach P, Buhler M, Von Der Goltz C, Mutschler J, Loeber S et al. Association of leptin with food cue-induced activation in human reward pathways. Archives of General Psychiatry 201269 529-537. (doi:10.1001/ archgenpsychiatry.2011.1586)

57 Batterham RL, Ffytche DH, Rosenthal JM, Zelaya FO, Barker GJ, Withers DJ \& Williams SC. PYY modulation of cortical and hypothalamic brain areas predicts feeding behaviour in humans. Nature 2007450 106-109. (doi:10.1038/nature06212)

58 De Silva A, Salem V, Long CJ, Makwana A, Newbould RD, Rabiner EA, Ghatei MA, Bloom SR, Matthews PM, Beaver JD et al. The gut hormones PYY 3-36 and GLP-1 7-36 amide reduce food intake and modulate brain activity in appetite centers in humans. Cell Metabolism $2011 \mathbf{1 4}$ 700-706. (doi:10.1016/j.cmet.2011.09.010)

59 Malik S, McGlone F, Bedrossian D \& Dagher A. Ghrelin modulates brain activity in areas that control appetitive behavior. Cell Metabolism 2008 7 400-409. (doi:10.1016/j.cmet.2008.03.007)

60 Goldstone AP, Prechtl CG, Scholtz S, Miras AD, Chhina N, Durighel G, Deliran SS, Beckmann C, Ghatei MA, Ashby DR et al. Ghrelin mimics fasting to enhance human hedonic, orbitofrontal cortex, and hippocampal responses to food. American Journal of Clinical Nutrition 201499 1319-1330. (doi:10.3945/ajcn.113.075291)

61 Van Oudenhove L, McKie S, Lassman D, Uddin B, Paine P, Coen S, Gregory L, Tack J \& Aziz Q. Fatty acid-induced gut-brain signaling attenuates neural and behavioral effects of sad emotion in humans. Journal of Clinical Investigation 2011121 3094-3099. (doi:10.1172/ JCI46380)

62 Kroemer NB, Krebs L, Kobiella A, Grimm O, Pilhatsch M, Bidlingmaier M, Zimmermann US \& Smolka MN. Fasting levels of ghrelin covary with the brain response to food pictures. Addiction Biology 201318 855-862. (doi:10.1111/j.1369-1600.2012.00489.x)

63 Li J, An R, Zhang Y, Li X \& Wang S. Correlations of macronutrientinduced functional magnetic resonance imaging signal changes in human brain and gut hormone responses. American Journal of Clinical Nutrition 201296 275-282. (doi:10.3945/ajcn.112.037440)

64 van Bloemendaal L, IJzerman RG, Ten Kulve JS, Barkhof F, Konrad RJ, Drent ML, Veltman DJ \& Diamant M. GLP-1 receptor activation modulates appetite- and reward-related brain areas in humans. Diabetes 201463 4186-4196. (doi:10.2337/db14-0849)

65 Akkary E. Bariatric surgery evolution from the malabsorptive to the hormonal era. Obesity Surgery 201222 827-831. (doi:10.1007/s11695012-0623-2)

66 Bueter M, Miras AD, Chichger H, Fenske W, Ghatei MA, Bloom SR, Unwin RJ, Lutz TA, Spector AC \& le Roux CW. Alterations of sucrose preference after Roux-en-Y gastric bypass. Physiology \& Behavior 2011 104 709-721. (doi:10.1016/j.physbeh.2011.07.025)

67 van de Sande-Lee S, Pereira FR, Cintra DE, Fernandes PT, Cardoso AR, Garlipp CR, Chaim EA, Pareja JC, Geloneze B, Li LM et al. Partial reversibility of hypothalamic dysfunction and changes in brain activity after body mass reduction in obese subjects. Diabetes 201160 1699-1704. (doi:10.2337/db10-1614)

68 Ochner CN, Kwok Y, Conceicao E, Pantazatos SP, Puma LM, Carnell S, Teixera J, Hirsch J \& Geliebter A. Selective reduction in neural responses to high calorie foods following gastric bypass surgery. Annals of Surgery 2011253 502-507. (doi:10.1097/SLA.0b013e318203a289)

69 Ochner CN, Stice E, Hutchins E, Afifi L, Geliebter A, Hirsch J \& Teixera J. Relation between changes in neural responsivity and reductions in desire to eat high-calorie foods following gastric bypass surgery. Neuroscience 2012209 128-135. (doi:10.1016/j.neuroscience.2012.02.030)

70 Bruce AS, Bruce JM, Ness AR, Lepping RJ, Malley S, Hancock L, Powell J, Patrician TM, Breslin FJ, Martin LE et al. A comparison of functional brain changes associated with surgical versus behavioral weight loss. Obesity 201422 337-343. (doi:10.1002/oby.20630)

71 Dunn JP, Cowan RL, Volkow ND, Feurer ID, Li R, Williams DB, Kessler RM \& Abumrad NN. Decreased dopamine type 2 receptor availability after bariatric surgery: preliminary findings. Brain Research 20102 123-130. (doi:10.1016/j.brainres.2010.03.064)

72 Scholtz S, Miras AD, Chhina N, Prechtl CG, Sleeth ML, Daud NM, Ismail NA, Durighel G, Ahmed AR, Olbers T et al. Obese patients after gastric bypass surgery have lower brain-hedonic responses to food than after gastric banding. Gut 201463 891-902. (doi:10.1136/gutjnl2013-305008)
Received 20 August 2014

Revised version received 12 March 2015

Accepted 8 April 2015 